\title{
The role of the emergency department in the acute management of chronic or recurrent pain
}

\author{
Diane MCLeod, RCpN, MN(Cl) ${ }^{a}$ * \\ Katherine Nelson, PhD, RN ${ }^{b}$
}

\author{
a Emergency Department, Nelson Public Hospital, Nelson, New Zealand \\ b Graduate School of Nursing, Midwifery \& Health (GSNMH), Victoria University of Wellington, New Zealand
}

Received 6 September 2012; received in revised form 5 December 2012; accepted 5 December 2012

\author{
KEYWORDS \\ Chronic nonmalignant \\ pain; \\ Emergency \\ department; \\ Emergency \\ management; \\ Persistent pain
}

\begin{abstract}
Summary
Background: Exacerbation of chronic pain is a common presenting problem for patients attending emergency departments (EDs), with many making multiple visits.

Aim: This paper aims to identify the role of the ED in the acute management of patients with persistent or chronic nonmalignant pain through a review of current literature.

Method: Four databases were searched using the MeSH and subject search terms "chronic nonmalignant pain", "persistent pain" and "emergency" and synonyms associated with these terms. Literature related to the underlying causes of suboptimal chronic pain management along with the sequelae associated with treatment or management was extracted.

Results: Three main themes emerged: patient expectations and satisfaction, barriers to care, and strategies/principles to improve ED management for this patient group. The presence of these themes appears to be partially due to time limitations for chronic issues in an acute department, accompanied by a lack of clear guidelines.

Conclusion: It is evident that the ED is not the ideal setting for managing patients with chronic pain however it is the last resort for many who do present, and who will continue to present should their pain persist. It is time to ensure that the ED provides a consistently supportive, cohesive and integrated approach to managing patients with chronic pain syndromes.

(c) 2013 College of Emergency Nursing Australasia Ltd. Published by Elsevier Ltd. All rights reserved.
\end{abstract}

\footnotetext{
* Corresponding author at: Emergency Department, Nelson Hospital, Private Bag 18, Tipahi St., Nelson 7042, New Zealand. Tel.: +64 03 5461800x7229; fax: +64035461961.

E-mail address: diane.mcleod@nmhs.govt.nz (D. McLeod).
}

\section{Introduction}

Chronic nonmalignant pain is highly prevalent throughout developed countries and is a common reason for patient presentation to the emergency department (ED)..$^{1-6}$ The International Association for the Study of Pain (IASP) ${ }^{7}$ defines this type of chronic pain as "pain that persists past the

1574-6267/\$ - see front matter @ 2013 College of Emergency Nursing Australasia Ltd. Published by Elsevier Ltd. All rights reserved. http://dx.doi.org/10.1016/j.aenj.2012.12.001 


\section{What is known}

- Exacerbation of chronic pain is a common reason for patient presentation to the emergency department. Many of these patients, with their complex needs, make multiple presentations despite the ED not being the ideal setting to manage their pain. This can cause distress and dissatisfaction for both the patients, and the ED clinicians (medical and nursing staff).

\section{What this paper adds}

- This literature review aims to clarify what is reported about the current roles of ED medical and nursing staff in the acute management of patients with chronic pain. There is a specific focus on issues that can cause patient and practitioner distress and dissatisfaction, and areas of inconsistencies and success in patient care.

- A number of strategies are identified that may improve management for this specific group. These include early identification of patients who have the potential to become one that presents multiple times (for example those who have presented four times or more for pain over a defined period of time). Further development of individualised plans and evidence based guidelines could improve both patient and clinician satisfaction through providing consistency of care. Ensuring an integrative approach involving the primary care provider, specialist pain team, the ED staff and the patient is critical to positive outcomes. A clinical nurse specialist and/or social workers within the ED seem ideally placed to coordinate this liaison. Supportive relevant education for nursing and medical staff will build knowledge, relieve uncertainties and alter existing attitudes in this specialist area.

- Scope for research arises around the value of nursing input with patients who present with chronic pain, as little specific information arises from the literature. Other areas for further research include patient presentation times, services already utilised and evaluation/audit of current practice and strategies utilised.

normal time of healing." It is a multi-faceted condition that can cause great distress, suffering and impact on the quality of daily life, activity and relationships of patients and their families. ${ }^{3,4,8}$ Four entities - the disease, the illness, the suffering and the pain behaviours - are aptly described as making up the complex matrix of chronic pain. 9,10

Patients with chronic pain account for some $12-16 \%$ of total ED visits, 1,11 with around $7 \%$ of people with chronic pain presenting multiple times. ${ }^{12}$ Despite the prevalence, this patient group with complex needs is often inadequately managed within ED. ${ }^{13}$ Numerous issues have been identified within the literature that can cause patient and practitioner dissatisfaction and distress. ${ }^{14-17}$ Despite these issues and frustrations, EDs are identified as having an important role in the care of patients with chronic pain. ${ }^{18}$ This role is likely due to the "all hours" availability of ED, the alternate option when the patient may be dissatisfied with primary care treatment, and the primary presentation scenario.

This review aims to clarify what is reported in the literature about the current role of ED in the acute management of patients with chronic pain. A specific focus is on issues that can cause patient and practitioner dissatisfaction and distress, and areas of success and inconsistency in patient care. Examination of these issues is important for informing service delivery and identifying areas for future research.

\section{Search strategy/methods}

PubMed, CINAHL, PsycINFO and Cochrane databases were searched with no date restrictions. Medical Subject Headings (MeSH) and subject terms used were ("chronic pain" OR "persistent pain" OR "chronic nonmalignant pain") combined with a search using the terms ("emergency department" OR "ED" OR "emergency room"' OR "acute care"' OR "accident and emergency"' OR "emergency management"). Truncated terms "rescue*", OR "breakthrough*" OR flare*" were also used. Searches were limited to humans and adults up to July 2011. Articles of all methodologies were considered. Reference lists were reviewed for other papers. Papers were included if they had a primary focus on the acute management of chronic pain in relation to the ED setting, and were excluded if they related only to acute pain, or to chronic malignant pain. Papers which covered presentations for both acute and chronic pain were included only if the material included provided additional information of relevance to chronic pain. Papers were read, critiqued and data extracted in relation to management and chronic pain. Twenty nine articles plus sections from one book were identified that contributed to the review. The Critical Appraisal Skills Programme (CASP) tools ${ }^{19}$ were used to assess systematic reviews $(n=1)$, randomised controlled trials $(n=1)$, and qualitative and quantitative studies $(n=13)$. The Woolliams et al. ${ }^{20}$ tool was used to consider the value of case studies and practice papers such as expert clinical discussions $(n=14)$. As this review utilised an integrative process, papers are referred to with respect to their particular contributions and not to the whole contribution their research made.

\section{Findings}

Of the 29 articles, 18 were directly linked with ED presentation or management, 4 were from the primary care setting and 7 in specific pain management areas with a focus on implications and linkages with emergency management. The research was conducted in four countries (USA 18, Canada 3, Australia 4, Sweden 1). Papers from 2005 to 2011 were predominantly from USA, probably reflecting the impact of the "Decade of Pain Control and Research" initiative. Authors included emergency clinicians and nurses, pain specialists including nurses, physicians and anaesthetists, psychologists 
and a pharmacologist. Each profession's observations, findings and opinions were from a slightly different lens, but congruent themes emerged that were integrated to give a comprehensive view of the needs and management of people with chronic pain in ED.

The CASP assessment found most studies were robust and provided descriptions of definitions, data extraction and analysis techniques. Some articles lacked a detailed description of the ED, missing data were not always accounted for and funding sources were not always named. Some studies reported use of more than one ED but did not take this into account in the analyses.

\section{Defining and describing chronic or persistent pain and the people who present with this}

There was no one definition of chronic pain, with the terms "chronic nonmalignant" and "persistent" being used interchangeably. There was also a divergence about how long a person should have pain before being defined as having chronic pain. Timeframes varied from 1 to 3 months ${ }^{1,7,21}$ to 6 months or more. ${ }^{3,22}$

In providing the definition of chronic pain, some authors differentiated it from acute pain by explaining that with chronic pain there is an inability of a person's body to restore its original physiological functions to normal homeostasis. It is not all "in the mind", arising from actual damage or dysregulation within the connective tissue (nociceptive) or within the pain neural system (neuropathic). ${ }^{9,23}$ Rather, the patient with chronic pain has a persistent sensitised state causing a hyper responsiveness to noxious (hyperalgesia) and non-noxious (ollodynia) stimuli that may cause a dramatic response and then may not be completely relieved or resolved. ${ }^{24}$

There is limited information available on whom the people are who present to the ED with chronic pain. People tend to be middle aged or older with a similar male to female ratio. ${ }^{5,11}$ Socioeconomic factors such as unemployment play a role for some of these patients. ${ }^{11}$ People with chronic pain have a mixed picture of general health issues, disability and past history with evidence emerging of high primary care hospital service and emergency department use. ${ }^{6,11}$ Blyth et al. $^{6}$ reported that from a community sample of people with chronic pain, attendance at ED ranged from 0 to 150 presentations over 12 months with some people admitted up to 20 times. Studies that collected data directly from people with chronic pain excluded those who were seriously unwell or hospitalised at time of presentation, or were unable to speak selected languages. Therefore, what is known from patient perspectives is mainly from a subset of people with chronic pain.

Exacerbations of chronic pain are referred to as "break through pain" or "flare ups". Pain pattern behaviours tend to be predictable and repetitive. ${ }^{23}$ Baker $^{23}$ describes the pervasiveness of an acute exacerbation of chronic pain being similar in many ways to a seizure disorder or cardiac presentation. Clinicians' attitudes vary between acute medical and chronic pain presentations despite treatment modalities overlapping to a degree in that treatment for chronic pain also follows a fairly expected or standard regime.

\section{Presenting to the ED with chronic pain}

People make a decision to present to the ED because of an exacerbation of worsening pain, an inadequate repertoire of coping strategies, disease severity and pure desperation with associated stress and anxiety. ${ }^{17,25-27}$ There is no clear identifiable cause of the chronic pain. Factors including sleep disturbance, psychological distress, social or vocational issues, illness behaviour, reduced activity, repeated treatment failures, complex medication use, disease progression, cultural issues or the constant desire to find a cure for the pain being the underlying causes of exacerbations. ${ }^{26}$ The most common presenting complaints are abdominal/flank pain, low back pain, headaches and chest pain. ${ }^{27,28}$ While some patients perceived the severity of symptoms as a threat to life or to personal autonomy, they were also aware that their own perception of urgency might differ from that of ED staff resulting in subsequent feelings of shame, loss of self esteem and increased dissatisfaction. ${ }^{29}$

On arrival at ED a primary expectation of the presenting patient is for expedient delivery of care, including analgesia and relief of pain. ${ }^{17,30}$ If this expectation is not met, or is delayed, patients can become distressed with behaviour indicative of dissatisfaction. This sometimes leads to patient complaints. ${ }^{1,31,32}$ In the study by Fosnocht et al. ${ }^{30}$ which included patients who presented with acute and chronic pain, patients reported a mean of $23 \mathrm{~min}$ as being an acceptable time to wait for analgesia compared with an actual time of $78 \mathrm{~min}$. A thorough physical examination, regardless of the number of previous presentations, is essential. It is beneficial to include assessment of the patient's risk profile in this. ${ }^{28,33}$ The risk profile is developed from examining precipitating factors such as low IQ, history of depression and exposure to family abuse against precipitating and perpetuating factors. Precipitating factors may be a minor fall, stress, or anxiety related to social or vocational issues. Combining these findings with perpetuating factors such as obesity, poor coping skills and financial issues helps to define areas to explore using a biopsychosocial approach. This can be reassuring for both patient and clinician, but is a somewhat lengthy approach given time limitations within the ED environment. Baker ${ }^{33}$ states that it is important to emphasise to the patient that severe pain does not necessarily predict tissue damage. Pain assessment is a crucial component of the cluster of vital signs gathered when triaging all patients presenting to ED. ${ }^{10,32}$ Distinguishing between legitimate pain and fraudulent presentations, addiction and pseudoaddiction is a concern for many clinicians. ${ }^{16,18,25,33}$

\section{Researched approaches for management of people who experience chronic pain}

Several approaches to ED care of patients with chronic pain have been trialled, mainly through concern about frequent presentations and potential abuse of medications. ${ }^{12,13,34,35}$ MacLeod and Swanson's ${ }^{13}$ coordinated care approach for patients with chronic pain aimed to minimise people presenting frequently to four EDs in Calgary, Canada. Strategies included tight control of medications dispensed, particularly opioids, along with improved communication and increased responsibility being laid on the primary care 
provider. Deterring drug seekers was an underlying theme, a label often mistakenly applied to patients with chronic pain. Unfortunately outcomes of this proposed system have not been published.

Treatment guidelines to limit opioid use were also described in a case report of two rural EDs by Hawkins et al. ${ }^{34}$ Subjective results showed that systematically limiting opioids through consistent, clear guidelines did not result in decreased patient satisfaction, and that both patient and staff satisfaction increased overall. Inclusion of statistical data would have strengthened this report.

Non-narcotic protocols aimed at reducing the need to present to the ED were trialled by Svenson and Meyer. ${ }^{12}$ Fifteen people presenting frequently (10 or more times) with chronic pain were enrolled in a prospective observational study. Treatment plan recommendations included using one practitioner only for each patient's treatment, limiting narcotic use, and recognising that escalating requirements for narcotics indicated the need for review of treatment. Although a small study, results were promising with a significant decline in ED presentations from an average of 19 to two visits per year. This suggests that non-narcotic policies may be a viable strategy for reducing frequent pain-related visits to EDs.

A behavioural health intervention, with the goals of increasing coping skills and enhancing motivation for change, achieved some success in reducing visits for high users of ED services. ${ }^{35}$ This may have been related to the accompanying use of disincentive graduated letters to the primary care provider. While the intervention was scientifically grounded, with benefits for both ED and the patient, the ready availability of behavioural health specialists within the department was required.

$A$ randomised control trial, based in primary care ${ }^{21}$ in USA, utilising similar collaborative interventions of clinician and patient education, pain treatment and individualised functional goals versus "treatment as usual" resulted in modest but statistically significant improvements. These improvements were in areas of self-reported pain related disability, pain intensity and depression severity. However, the funding required to introduce this collaborative approach may not be affordable for many EDs.

\section{Recommended strategies and guidelines for patients with chronic pain}

A number of authors suggest strategies and guidelines to improve acute management in ED. ${ }^{10,13,17,18,32-34}$ The importance of initial assessment followed by multimodal drug therapy combined with a biopsychosocial approach is advocated and has demonstrated increasing promise for those with chronic pain. This approach is supported by primary care recommendations and guidelines with the acknowledgement that implementation may be difficult in practice due to the complex nature of chronic pain. ${ }^{10,21,26,28,33}$ Although some of these studies are based in primary care, several principles and strategies can be applied to the ED setting.

Following assessment, documented monitoring of pain and functional levels should take place on a regular basis using validated and appropriate pain assessment tools. ${ }^{25}$
Ducharme $^{25}$ states there is no reliable way of objectively measuring pain, with chronic pain scales being impractical for ED use. The $0-10$ pain scale is accepted practice in most EDs and is familiar to patients. Its use is recommended in the literature, aiming for a 2 out of 10 reduction in pain, or $30 \%$ improvement in functional level for those with chronic pain. ${ }^{10,22}$ Determining an acceptable level of tolerable pain for each patient is important. What is acceptable for one, may not be for another. ${ }^{22}$ From examining the literature it seems reasonable and realistic to aim for a reduction in pain level, as opposed to total relief, along with improvement in function and minimisation of interference of pain in a person's lifestyle. ${ }^{26}$ Patient expectations should therefore be altered from "cure" to "coping" or "living with less pain'. Assessment of anxiety levels must also be considered and treated appropriately. ${ }^{32}$ This is important because people with chronic pain are exquisitely sensitive to cognitive and verbal reinforcement, requiring a consistent approach by ED staff. ${ }^{18,22,33}$ Concerns must be addressed to avoid simplistic reassurance or denial of disease. Similarly ED staff should not overemphasise medications or testing. Both tend to reinforce that the pain experienced is purely physical.

Multimodal drug therapies are often required for complex disease states, such as heart disease and diabetes, and can be rationalised for treatment of chronic pain with its neurobiological complexities. ${ }^{10,15}$ Baker and others ${ }^{17,18,32,33}$ take the stance that definitive treatment of chronic pain is not the role of the ED, but rescue medication, rational investigation and referrals are appropriate. There is also agreement that rescue medication should include an appropriate multimodal selection of paracetemol, nonsteroidal anti-inflammatory drugs, tramadol, ketamine and local anaesthetics. ${ }^{24,33,34}$ The addition of narcotics remains controversial. Some authors justify use of opioids, ${ }^{24,33}$ others oppose, ${ }^{12,18,34}$ and those in the middle advocate cautious consideration. ${ }^{7,36}$ Consideration must also be given to the patient's usual analgesic regime taking care not to initiate or exacerbate psychological or tolerance related complications of chronic pain. ${ }^{18}$ Meeting analgesic needs correlated closely with patient satisfaction as did an "expression of caring". These findings were validated in a qualitative analysis within primary care highlighting the importance of a "listening ear', trust and accessibility to clinician care. ${ }^{15}$ In a study by Todd et al. ${ }^{17}$ factors influencing satisfaction were similar and included wait time, effectiveness of analgesia, education specific to pain, specialist referral and being treated with dignity and respect. It is of concern that $11 \%$ of participants in the study stated treatment provided by ED staff made them feel like "drug seekers."

Targeted medication guidelines have been developed for specific pain presentations by expert clinicians. ${ }^{7,10,18,24,32,34}$ These provide for consistency of care using predominantly non opioid medication regimes for complaints such as migraines, back pain, toothache and neuropathic pain. Regimes have resulted from neurobiological research that has identified detailed therapy that can be directed to specific pain receptors with positive effect. Emergency physicians' perception of quality of care delivered as well as job satisfaction increased after implementation of guidelines in one study ${ }^{34}$ demonstrating that sound evidence based guidelines are well worthwhile adopting. 
Caution regarding the prescribing of habit forming medications and, in particular, oxycontin is raised by Hawkins et al. ${ }^{34}$ Gouke $^{24}$ makes pertinent comment regarding safety with opioid use, recommending using only one doctor for supply of medication, particularly opioids, and educating patients to accept responsibility for ensuring their supply of medications does not run out after hours.

Non-drug interventions are equally important in relieving the stress and suffering of the chronic pain experience. ${ }^{10,17,21,24,26,32,33}$ While some interventions such as relaxation techniques and patient education may be discussed within the emergency department, referral to a pain service and specialists within the multidisciplinary team to address specific needs and consolidate coping skills is essential. ${ }^{25,28,34}$ Strategies should be selected according to needs, based on the cognitive, behavioural and emotional state of the patient and must be in consultation with the patient. ${ }^{26,27}$ These may include exercises, activity-paced strategies, biofeedback, relaxation techniques, assertiveness skills training, and dealing with "flare-ups" without resorting to medication alone. This is a complex process to be undertaken with adequate time and expertise. The New Zealand Accident Compensation Corporations guidelines ${ }^{26}$ make the salient point that the patient must do most of the work to stay well, and to keep doing it. Health professionals must provide support and encouragement to promote self-management without creating dependency.

Individualised up-to-date management plans are a valuable tool for all patients with complex needs as they can assist with prompt initiation of assessment and treatment by having basic information and past history succinctly documented and available on patient presentation. ${ }^{15,27}$ Clinicians are then able to focus on the current presentation and patient needs with consistency of approach and care. ${ }^{27,37}$ Development of care plans are best undertaken through a multidisciplinary approach involving first and foremost the patient, the primary care provider, pain specialist teams, ED physicians and specialist nurses. ${ }^{22,37}$

Following every patient presentation, a follow up letter should go to the patient's primary care physician to maintain continuity and dialogue about the patient. ${ }^{13,18,22,32}$ The patient should receive a copy of any such correspondence to maintain full involvement, trust and respect for the professional team.

Finally, quality assurance and quality improvement strategies are discussed as important aspects of the ED pain control programme. ${ }^{16,32}$ Strategies include use of quality indicators, adopting the use of electronic records or a prescription monitoring service, feedback of audits on practice to staff and ongoing staff education. Such activities are important in embedding and improving evidence based practice within ED, especially in an area such as chronic pain management that is prone to confusion and inconsistencies.

\section{Issues with managing chronic pain in the ED environment}

The majority of authors emphasise that the ED is not the ideal setting for the management of complex chronic pain issues. ${ }^{11,13,18,33}$ Articles over the last 20 years confirm and reflect on challenges and complexities that can cause patient and practitioner dissatisfaction and distress by posing barriers to optimal care.

In 2008, Wilsey and colleagues conducted two qualitative studies in the US to examine attitudes and barriers to chronic pain management in ED. In the first study ${ }^{14} 24$ physicians were surveyed. The main barriers identified were time limitations for consultation and low triage priority, with wait times further delayed by acute life threatening presentations. These were classified as system issues. Other system-related barriers such as lack of a primary care physician, financial issues, and "frequent flyers" were acknowledged. Physician attitudinal barriers included the "annoyance" factor, fear of labelling of a patient as "bad" or of having an addiction, concern regarding diversion of medications to the black market, and belief in the need of pathology to support a diagnosis. Educational needs for providers were not discussed but have been raised in other literature. ${ }^{11,31,38}$ Results of the second study ${ }^{39}$ revealed that patients were significantly more likely than providers to believe their pain needed to have a diagnosis to be treated. Practitioners were significantly more likely to believe that patients presented because of the lack of a primary care provider. Nurses, physicians and patients were in agreement that chronic pain treatment was not a priority in ED. Despite this notion, practitioners concluded that patients with exacerbations of chronic pain should be reassured that their pain will be treated, despite the absence of objective signs or magnified symptoms. Treatment recommendations are not always straight forward however.

The scientific evidence for escalating use and abuse of opioids is unclear, continuing to be the subject of ongoing discussion and study. ${ }^{36,39}$ The benefits and risks of prescribing opioids for chronic pain are questioned, with some emergency physicians rationalising a non-narcotic protocol for exacerbations of chronic pain. ${ }^{12,34}$ Others, however, argue opioids have a place in rescue therapy. ${ }^{22,33}$ Lack of coordinated care resulting potentially in increasing risk of abuse through having multiple prescribers is also raised. ${ }^{16,32}$ And then there is the issue of antisocial behaviour from substance users who may have complex psychosocial histories requiring increased attention from providers. ${ }^{40}$ The challenges of addressing and responding to the negative emotions, depression, anxiety and anger that a person with chronic pain often presents with must not be overlooked. This can be overwhelming for many ED staff to cope with within time constraints and without the coordinated team approach or longitudinal view of care. However, Peppin ${ }^{38}$ boldly states that "the realities of treating chronic pain do not reflect the attention that marginalisation of patients taking opioids has received"'. He claims that discussion around barriers to adequate pain treatment should also be considered as moral issues, and not exclusively rationalised as clinical aberrations. Peppin joins other clinicians in advocating for an altered perspective, seeking solutions and focusing on successes of treatment as opposed to barriers. ${ }^{21,32-35}$

\section{Discussion and recommendations}

It is apparent that patients with chronic non malignant pain will continue to present to EDs for pain management, 
particularly for rescue treatment. A number of factors arise as a result of this review that could improve management and subsequent satisfaction for both patient and clinicians.

The literature demonstrates the importance of ED physicians and nursing staff having both the knowledge to understand the neurophysiology of chronic pain along with the skills to manage the illness, distress and behaviours that frequently accompany chronic pain. While the task is challenging, being able to differentiate chronic pain exacerbations from acute intermittent pain and addictive behaviours would minimise some stress for clinicians. Additional educational opportunities may be required to address knowledge deficits or uncertainties regarding practice.

Acquisition of knowledge may be gained through the Faculty of Medicine and of Anaesthesia at some tertiary institutions who offer courses on chronic conditions and pain management. There are also some web based on-line courses available for both clinicians and patients. Direct teaching and resource support from pain specialists and anaesthetists with a special interest in chronic pain is worthwhile, as is taking the opportunity to observe the practice of pain services and other EDs. Building specific knowledge in this area may help relieve staff and patient frustrations and alter existing attitudes.

When considering reasons for patients with chronic pain presenting to EDs, data regarding the availability of the primary care provider and the actual time of presentation were not included in studies reviewed. If further study shows that patients present predominantly after hours, other strategies such proposals for additional specialist staff should be examined.

Appropriate treatment and principles of care should be clearly outlined in evidence based guidelines, protocols, or individualised care plans. It is important to identify people who have the potential to become a person who presents on multiple occasions as early as possible. For example anyone who has presented four or more times for pain should be referred to a pain service and a management plan negotiated for how best to manage exacerbations in the community setting before coming to ED. This is accepted practice for other chronic conditions such as asthma, angina and anxiety disorders. Applying the strategies concerning assessment, management and follow-up as described in the literature should be used where possible. The current lack of evaluative studies on interventions needs to be addressed, but should not deter changes in practice meanwhile.

An integrated approach to treatment is critical. Open and regular communication with the primary care provider, specialist pain team, the patient and the ED are crucial to successful and improved management for this patient group. Patient "buy in" and cooperation appear to increase when they are aware of the plan and have had input into it. Enhanced communication can then occur between all services involved leading to positive outcomes for the patient. A clinical nurse specialist or social workers within ED seems ideally placed to coordinate this liaison, and ensure individual care plans are developed, updated and relevant. ${ }^{22,37}$ The potential for this role requires exploration but could provide benefits for both the ED and the patients. Despite nurses being the largest workforce in most EDs little is known about the value of nursing input with patients with chronic pain. Emergency nurses have a crucial role to play in patient care, education and support and the extent of their involvement in all aspects of care and how such involvement can be enhanced needs to be understood.

The ongoing debate regarding the use and abuse of opioids requires further study and research. Sound scientific recommendations and non opioid drug alternative therapies are required. These should include current prescribing practice especially for drugs such as oxynorm, with increasing concern over its addictive qualities and abuse worldwide.

Chronic pain requires evaluation and treatment that differs greatly from acute pain. However, the connection between poorly managed acute pain and possible development of chronic pain needs to be made well known and understood by ED clinicians and other health practitioners. There is increasing evidence that appropriate early aggressive management of acute pain will minimise the transition to persistent pain. ${ }^{23-25,41}$ Those patients at risk of developing chronic pain can display early behaviours such as reacting to acute pain by "catastrophising"' events. Addressing such responses to pain at the acute stage may forestall development of this distressing condition.

\section{Conclusion}

This review of literature confirms that the ED role is clearly not the definitive treatment of chronic non malignant pain. Never the less, EDs will be required to continue to make rescue treatment and care available for individuals who are disconnected from other health care providers. ED staff must ensure their practices contribute to the solution, and not the problems associated with chronic pain. People with chronic pain need non-judgmental support that is available around the clock. Currently there are limited, or a complete absence of, services outside the ED in many regions. An altered perspective focusing on solutions and successes of treatment modalities is essential to ensure this patient group, who could be perceived as being marginalised, is treated with the same respect as other patients presenting with recurrent chronic health problems.

\section{Contributions}

Diane McLeod reviewed articles and was lead author of paper. Katherine Nelson contributed to paper development and final paper.

\section{Provenance and conflict of interest}

None declared. This paper was not commissioned.

\section{Funding}

Nil.

\section{References}

1. Neighbor M, Puntillo K, Homel P, Todd K. Chronic pain in the emergency department. Acad Emerg Med J 2007;14(Suppl.):S112. 
2. Cordell W, Keene K, Giles B. The high prevalence of pain in emergency medical care. Am J Emerg Med 2002;20(3):165-9.

3. Gureje O, Von Korff M, Simon G, Gater R. Persistent pain and well-being: A World Health Organization Study in Primary Care. J Am Med Assoc 1998;280(2):147-51.

4. Breivik H, Collett B, Ventafridda V, Cohen R, Gallacher D. Survey of chronic pain in Europe: prevalence, impact on daily life, and treatment. Eur J Pain 2006;10(4):287-333.

5. Blyth F, March L, Brnabic A, Jorm L, Williamson M, Cousins M. Chronic pain in Australia: a prevalence study. Pain 2001;89(2-3):127-34.

6. Blyth F, March L, Brnabic A, Cousins M. Chronic pain and frequent use of health care. Pain 2004;111(1-2):51-8.

7. International Association for the Study of Pain (ISAP). Classification of chronic pain. Description of chronic pain syndromes and definition of pain terms. Pain 1986;(Suppl. 3):S1-226.

8. Hanley $\mathrm{O}$, Miner J, Rockswold E, Biros M. The relationship between chronic illness, chronic pain, and socioeconomic factors in the ED. Am J Emerg Med 2011;29(3):286-92.

9. Loeser J, Melzack R. Pain: an overview. Lancet 1999;353(9164):1607-9.

10. Argoff C, Albrecht P, Irving G, Rice F. Multimodal analgesia for chronic pain: rationale and future directions. Pain Med 2009;10(S2):S53-66.

11. Bernard A, Wright S. Chronic pain in the ED. Am J Emerg Med 2004;22(6):444-7.

12. Svenson J, Meyer T. Effectiveness of nonnarcotic protocol for the treatment of acute exacerbations of chronic nonmalignant pain. Am J Emerg Med 2007;25(4):445-9.

13. MacLeod D, Swanson R. A new approach to chronic pain in the ED. Am J Emerg Med 1996;14(3):323-6.

14. Wilsey B, Fishman S, Crandall M, Casamalhuapa C, Bertakis K. A qualitative study of the barriers to chronic pain management in the ED. Am J Emerg Med 2008;26(3):255-63.

15. Upshur CC, Bacigalupe G, Luckmann R. They don't want anything to do with you'": patient views of primary care management of chronic pain. Pain Med 2010;11(12):1791-8.

16. Jan S. Patient perspective, complexities, and challenges in managed care. J Manag Care Pharm 2010;16(Suppl. 1-b):S22-5.

17. Todd K, Cowan P, Kelly N, Homel P. Chronic or recurrent pain in the emergency department: national telephone survey of patient experience. West J Emerg Med 2010;XI(5):408-15.

18. Hansen $G$. Management of chronic pain in the acute care setting. Emerg Med Clin North Am 2005;23(2):307-38.

19. Oxford University Public Health Resources Unit. Appraisal tools; 2007. Available from: http://www.phru.nhs.uk/Pages/PHD/ resources.htm

20. Woolliams M, Williams K, Butcher D, Pye J. Be more critical. A practical guide for health and social care; 2009. Available from http://www.brookes.ac.uk/services/upgrade/pdf/ Bemorecritical2.pdf

21. Dobscha S, Corson K, Perrin N, Hanson G, Leibowitz R, Doak M, et al. Collaborative care for chronic pain in primary care. $\mathrm{J} \mathrm{Am}$ Med Assoc 2009;301(12):1242-52.
22. McCaffrey M, Pasero C. Pain: clinical manual. 2nd ed. St. Louis: Mosby; 1999.

23. Baker K. Recent advances in the neurophysiology of chronic pain. Emerg Med Australas 2005; 17:65-72.

24. Gouke C. The management of persistent pain. Med J Aust 2003;178(9):444-7.

25. Ducharme J. Acute pain and pain control: state of the art. Ann Emerg Med 2000;35(6):592-603.

26. ACC. What is pain management for persistent pain? ACC Development Unit 2007. Report No.: ACC2651 Contract No.: ACC2651.

27. Milbrett P, Halm M. Characteristics and predictors of frequent utilization of emergency services. J Emerg Nurs 2009;35(3):191-8.

28. Disorbio J, Bruns D, Barolat G. Assessment and treatment of chronic pain: a physicians guide to a biopsychosocial approach. Pract Pain Manag 2006;6(2):1-10.

29. Olsson M, Hansagi $H$. Repeated use of the emergency department: qualitative study of the patient's perspective. Emerg Med J 2001;18(6):430-4.

30. Fosnocht D, Swanson E, Bossart P. Patient expectations for medication delivery. Am J Emerg Med 2001;19(5):399-402.

31. Fosnocht $D$, Swanson $E$, Barton $E$. Changing attitudes about pain and pain control in emergency medicine. Emerg Med Clin North Am 2005;23:297-306.

32. Richards C. Establishing an emergency department pain management system. Emerg Med Clin North Am 2005;23(2): 519-27.

33. Baker K. Chronic pain syndromes in the emergency department: identifying guidelines for management. Emerg Med Australas 2005; 17(1):57-64.

34. Hawkins S, Smeeks F, Hamel J. Emergency management of chronic pain and drug-seeking behaviour: an alternate perspective. J Emerg Med 2008;34(2):125-9.

35. Woodhouse J, Peterson M, Campbell C, Gathercoal K. The efficacy of a brief behavioral health intervention for managing high utilization of ED services by chronic pain patients. J Emerg Nurs 2010;36(5):399-403.

36. Manchikanti L, Fellows B, Ailinani H, Pampati V. Therapeutic use, abuse, and nonmedical use of opioids: a ten-year perspective. Pain Physician 2010;13(5):401-35.

37. Pope D, Fernandes CM, Bouthillette F, Etherington J. Frequent users of the emergency department: a program to improve care and reduce visits. Can Med Assoc J 2000;162(7): 1017-20.

38. Peppin J. The marginalization of chronic pain patients of chronic opioid therapy. Pain Physician 2009;12:493-8.

39. Wilsey B, Fishman S, Ogden C, Tsodikov A, Bertakis K. Chronic pain management in the emergency department: a survey of attitudes and beliefs. Pain Med 2008;9(8):1073-80.

40. Neighbor M, Dance T, Hawk M, Kohn M. Heightened pain perception in illicit substance-using patients in the ED: implications for management. Am J Emerg Med 2011;29:50-6.

41. Sinatra R. Causes and consequences of inadequate management of acute pain. Pain Med 2010;11(12):1859-71. 